\title{
ANALYSIS OF A MULTIACCESS CONTROL SCHEME ${ }^{1}$
}

John N. Tsitsiklis ${ }^{2}$

\begin{abstract}
We analyze a particular scheme for controlling a multiaccess channel. We prove that this scheme is stable and may achieve throughputs up to $1 / e$.
\end{abstract}

1. Research supported by the Army Research Office under Contract DAAAG-29-84-K-0005 and by the Office of Naval Research under Grant ONR/N00014-77-C-0532.

2. Laboratory for Information and Decision Systems, Massachusetts Institute of Technology, Cambridge, MA 02139. 


\section{DESCRIPTION OF THE CONTROL SCHEME.}

Consider the usual slotted ALOHA model, under the infinite source assumption and ternary feedback. In more detail, there is an infinite number of stations and, at the beginning of any time slot, each station may have at most one packet to transmit. Any station with an available packet may decide to attempt transmission (possibly using a probabilistic rule) or to decide to defer this attempt for later. Let $Y_{t}$ be the number of attempted transmissions during the $t$-th slot. If $Y_{t}=0$ we say that a "hole" has occured. If $Y_{t}=1$, the (single) attempted trasnmission is succesful. Finally, if $Y_{t} \geq 2$, there is a collision and no packet is succesfully transmitted. At the end of the $t$-th slot all stations learn whether a hole, a success, or a collision has occured. Accordingly, we define the variable $Z_{t}$ to be equal to $Y_{t}$, if $Y_{t}<2$, and equal to 2, if $Y_{t} \geq 2$. The information available to any station at the beginning of the $t$-th slot is the collection of variables $Z_{1}, \ldots, Z_{t-1}$. The decision of a station, whether it will attempt transmission during the $t$-th slot, is constrained to be a function of $Z_{1}, \ldots, Z_{t-1}$ and possibly an internal random number generator.

We assume that during the $t$-th slot a random number $A_{t}$ of new stations generate a packet which they would like to eventually transmit. We assume that the random variables $A_{t}$ are independent and identically distributed according to a Poisson distribution with mean $\lambda$. Let $N_{t}$ be the number of stations with a packet available for transmission at the beginning of the $t$-th slot. Then, $N_{t}$ evolves as follows: $N_{t+1}=N_{t}+A_{t}-1$, if $Z_{t}=1 ; N_{t+1}=N_{t}+A_{t}$, otherwise.

The objective is to find a probabilistic rule that lets each station decide at any given time, using only the information available to it, whether it will transmit or not. (Of course this rule will be used only by those stations that have an available packet.) This rule should be stable, that is, the stochastic process $N_{t}$ should not "explode, in a suitable mathematical sense. One particular scheme which accomplishes this objective was presented in [2-3].

Rivest [1] has suggested the following strategy. At the beginning of the $t$-th slot, each station has available the same estimate $\hat{N}_{t}$ of $N_{t}$. Each station with an available packet attempts transmission with probability $1 / \hat{N}_{t}$. Conditioned on $\hat{N}_{t}$, the decisions of different stations are statistically independent and independent of any other events that have occured in the past. (It is not hard to show that if $N_{t}$ is large and if $\hat{N}_{t}=N_{t}$, then the above choice of transmission probability is optimal, in the sense that it maximizes the probability of a succesful transmission during the $t$-th slot.) The novelty of the scheme lies in the procedure for updating the estimate $\hat{N}_{t}$, which is the following:

(i) If $Z_{t}<2$, then $\hat{N}_{t+1}=\max \left\{1, \hat{N}_{t}-1+\hat{\lambda}\right\}$;

(ii) If $Z_{t}=2$, then $\hat{N}_{t+1}=\hat{N}_{t}+\frac{1}{e-2}+\hat{\lambda}$.

In these equations, $\hat{\lambda}$ is an estimate of $\lambda$.

This updating procedure is motivated in [1] as an approximation of the exact Bayesian formula for updating the optimal estimate $E\left[N_{t} \mid Z_{0}, \ldots, Z_{t-1}\right]$. We define $X_{t}=\left(N_{t}, \hat{N}_{t}\right)$ and we notice that, for any fixed values of $\lambda$ and $\hat{\lambda}, X_{t}$ is a Markov process taking values in a countable state space, 
assuming that $\hat{N}$ is initialized with an integer value.

In this paper we analyze the stability of this scheme. Our main results can be summarized as follows:

(a) If $\lambda$ is known exactly, and therefore $\hat{\lambda}=\lambda$, then the Markov process $\left(N_{t}, \hat{N}_{t}\right)$ is geometrically ergodic if $\lambda<e^{-1}$. We should point out here that no control strategy in which all stations use the same probability of transmission could achieve throughput larger than or equal to $e^{-1}$ and, in this sense, the above scheme is optimal.

(b) If $\lambda$ is not known exactly, but rather an inexact estimate $\hat{\lambda}$ is used in the updating equations (1.1), (1.2), then the scheme is stable (geometrically ergodic) if $\lambda<e^{-1}, \hat{\lambda} \leq e^{-1}$ and $\lambda \leq \hat{\lambda}$. We also provide a heuristic argument which suggests that if $\hat{\lambda}<\lambda$ and if the difference between $\lambda$ and $\hat{\lambda}$ exceeds a certain threshold, then instability may result, even if $\lambda<e^{-1}$.

In [1], it is suggested that $\hat{\lambda}$ could be formed by estimating $\lambda$ on-line. In particular, one may let $\hat{\lambda}_{t}$, the estimate at time $t$, be equal to the number of succesful transmissions so far, divided by the time elapsed. Alternatively, one may use a sliding window, or discount past successes, so that the estimators $\hat{\lambda}_{t}$ retain their adaptivity, as $t \rightarrow \infty$. However, our results suggest that letting $\hat{\lambda}=e^{-1}$ may be an equally reasonable (and possibly more robust) alternative than on-line estimation of $\lambda$.

Reference [4] discusses a related class of schemes which have been introduced and analyzed in [5]. The discussion of [4] shows that the scheme introduced in [1], with $\hat{\lambda}=e^{-1}$, is a special case of those analyzed in [5] and that, in particular, it is known that the scheme is stable. In this light, the only contribution of this paper is a possibly new method for obtaining this convergence result. In fact, we have not yet had access to reference [5] to see whether the proof technique is different or not.

\section{MATHEMATICAL PRELIMINARIES.}

A random variable $W$ is exponential type if there exist $d>0, D$ such that $E\left[e^{d|W|}\right] \leq D$. Let $\left\{X_{k}\right\}$ be an irreducible aperiodic Markov chain on a countable state space. We say that $\left\{X_{k}\right\}$ is geometrically ergodic if there exists a state $x$ such that the stopping time $r=\min \left\{t>0: X_{t}=x\right\}$ is exponential type, for any initial state $X_{0}$.

Let $\left\{W_{t}\right\}$ be a sequence of random variables adapted to an increasing family $\left\{\mathcal{F}_{t}\right\}$ of $\sigma$-fields. We say that $\left\{W_{t}, \mathcal{F}_{t}\right\}$ is exponential type if there exist $d>0, D$, such that

$$
E\left[e^{d\left|W_{t+1}-W_{t}\right|} \mid F_{t}\right] \leq D, \quad \forall t \geq 0 .
$$

We will use the following result of [2].

Proposition 2.1: Suppose that $\left\{W_{t}, \mathcal{F}_{t}\right\}$ is exponential type and that for some $\epsilon>0, a \in \Re$, we have

$$
E\left[W_{t+1}-W_{t} ; W_{t}>a \mid \xi_{t}\right] \leq-\epsilon, \quad \forall t \geq 0 .
$$

Then the stopping time $\tau=\min \left\{t \geq 0: W_{t} \leq a\right\}$ is exponential type. 
We will also need the following result which is proved in a way similar to the results of [2].

Proposition 2.2: Let $\left\{W_{t}, \digamma_{t}\right\}$ be exponential type, with $W_{0}=0$, and let $J$ be a positive integer. Let $\tau$ be a stopping time (with respect to $\left\{\mathcal{F}_{t}\right\}$ ) and assume that there exists some $\epsilon>0$ such that $E\left[W_{t+1}-W_{t} ; \tau>t \mid F_{t}\right] \leq-\epsilon, \forall t$. Then there exists some $B$ (depending only on $d, D$, $\epsilon$, but not on $J$ or the statistics of $\tau$ ) such that $E\left[\max \left\{0, W_{J}\right\} ; \tau>J \mid F_{0}\right] \leq B$.

Proof: Since $\left\{W_{t}, \digamma_{t}\right\}$ is exponential type and using our assumption on $W_{t+1}-W_{t}$, there exists some $\eta>0$ (depending only on $d, D, \epsilon$ ) such that

$$
E\left[e^{\eta\left(W_{t+1}-W_{t}\right)} ; \tau>t \mid F_{t}\right] \leq 1, \quad \forall t
$$

(This is proved in Lemma 2.1 of [2].) We use the inequality $\max \{0, x\} \leq \frac{1}{\eta} e^{\eta x}, \forall x$, to obtain

$E\left[\max \left\{0, W_{J}\right\} ; \tau>J \mid \mathcal{F}_{0}\right]=E\left[\max \left\{0, W_{J \wedge \tau}\right\} ; \tau>J \mid \mathcal{F}_{0}\right] \leq \frac{1}{\eta} E\left[e^{\eta W_{J \wedge r}} ; \tau>J \mid \mathcal{F}_{0}\right] \leq \frac{1}{\eta} E\left[e^{\eta W_{J \wedge r}} \mid \mathcal{F}_{0}\right]$

We now notice that the stochastic process $e^{\eta W_{k \wedge r}}$ is a supermartingale, as a consequence of (2.3). Therefore, $E\left[e^{\eta W_{J \wedge r}}\right] \leq 1$, which gives the desired result with $B=\frac{1}{\eta}$.

\section{MAIN RESULT.}

Theorem 3.1: If $0<\hat{\lambda} \leq e^{-1}, 0<\lambda<e^{-1}$ and $\lambda \leq \hat{\lambda}$, then the Markov process $X_{t}$, defined in Section I, is geometrically ergodic.

Proof: We will be using the notation $\tilde{N}_{t}=\hat{N}_{t}-N_{t}$ and $\tilde{\lambda}=\hat{\lambda}-\lambda$. We also define $\mathcal{F}_{t}$ as the $\sigma$-field generated by $\left\{A_{n-1}, N_{s}, \hat{N}_{s}: s<t\right\}$. We start by establishing approximate formulas for the drift. of $N_{t}$ and $\tilde{N}_{t}$. We define two functions on the state space: $c(N, \hat{N})=E\left[N_{t+1}-N_{t} \mid X_{t}=(N, \hat{N})\right]$ and $d(N, \dot{N})=E\left[\tilde{N}_{t+1}-\tilde{N}_{t} \mid X_{t}=(N, \hat{N})\right]$. Using the binomial probability formulas, we obtain

$$
\begin{gathered}
c(N, \hat{N})=\lambda-\frac{N}{\hat{N}}\left(1-\frac{1}{\hat{N}}\right)^{N-1} \\
d(N, \hat{N})=\frac{1}{e-2}\left(1-\frac{N_{t}}{\hat{N}_{t}}\left(1-\frac{1}{\hat{N}}\right)^{N-1}-\left(1-\frac{1}{\hat{N}}\right)^{N}\right)-\left(1-\frac{1}{\hat{N}}\right)^{N}+\tilde{\lambda}
\end{gathered}
$$

We also introduce a function $f:[0, \infty) \times\left(0, e^{-1}\right] \mapsto \Re$, defined by

$$
f(\alpha, \tilde{\lambda})=\frac{1}{e-2}\left(1-\alpha e^{-\alpha}-e^{-\alpha}\right)-e^{-\alpha}+\tilde{\lambda}
$$

Lemma 3.1: There exists a function $h: \Re \mapsto \Re$ such that $\lim _{M \rightarrow \infty} h(M)=0$ and such that, if $N \geq M$ or $\hat{N} \geq M$, then

$$
\begin{gathered}
\left|c(N, \hat{N})-\left(\lambda-\alpha e^{-\alpha}\right)\right| \leq h(M), \\
|d(N, \hat{N})-f(\alpha, \tilde{\lambda})| \leq h(M),
\end{gathered}
$$


where $\alpha=\frac{N}{N}$.

Proof: (Outline) For any fixed value of $\alpha$, the result is immediate from the formulas (3.1), (3.2) and the fact $\lim _{\hat{N} \rightarrow \infty}\left(1-\frac{1}{\hat{N}}\right)^{\hat{N}}=e^{-1}$. The fact that the bounds are actually uniform over all $\alpha$ may be easily demonstrated by working out an exact expression for the approximation error, or by appealing to the similar bounds developed in [3].

We now study the properties of the function $f$.

Lemma 3.2: (i) For any $\tilde{\lambda}$, the function $f$ is strictly increasing in $\alpha$.

(ii) For any $\tilde{\lambda} \in\left(0, e^{-1}\right]$, there exists a unique $\alpha=g(\tilde{\lambda}) \in(0,1]$ such that $f(\alpha, \tilde{\lambda})=0$.

(iii) If $\lambda \in\left(0, e^{-1}\right) \cap(0, \hat{\lambda}]$, then $g(\tilde{\lambda}) e^{-g(\tilde{\lambda})}>\lambda$.

Proof:(i) This is implied by the inequality $\frac{\partial f}{\partial \alpha}(\alpha, \tilde{\lambda})=\frac{1}{e-2} \alpha e^{-\alpha}+e^{-\alpha}>0, \forall \alpha>0$.

(ii) Existence of a solution in the desired range follows from $f(0, \tilde{\lambda})=-1+\tilde{\lambda}<0, f(1, \tilde{\lambda})=\tilde{\lambda} \geq 0$ and the continuity of $f$. Uniqueness follows from the strict monotonicity of $f$.

(iii) Suppose first that $\lambda=\hat{\lambda}$. Then, $g(\tilde{\lambda})=g(0)=1$ and $g(0) e^{-g(0)}=e^{-1}>\lambda$, as desired. So suppose that $\lambda \neq \hat{\lambda}$. We use the equation $\frac{d f}{d \tilde{\lambda}}(g(\tilde{\lambda}), \tilde{\lambda})=0$, to obtain

$$
\frac{d g}{d \tilde{\lambda}}(\tilde{\lambda})=\frac{-1}{\frac{1}{e-2} g(\tilde{\lambda}) e^{-g(\tilde{\lambda})}+e^{-g(\tilde{\lambda})}} .
$$

Thus,

$$
\frac{d}{d \tilde{\lambda}}\left[g(\tilde{\lambda}) e^{-g(\tilde{\lambda})}\right]=[1-g(\tilde{\lambda})] e^{-g(\tilde{\lambda})} \frac{d g}{d \tilde{\lambda}}(\tilde{\lambda}) \geq \frac{-1}{\frac{1}{e-2} g(\tilde{\lambda})+1}>-1 .
$$

Hence, for $\tilde{\lambda}>0, g(\tilde{\lambda}) e^{-g(\bar{\lambda})}>g(0) e^{-g(0)}-\tilde{\lambda}=e^{-1}-\tilde{\lambda} \geq \hat{\lambda}-\tilde{\lambda}=\lambda$.

From now on we use $\beta$ to denote the value of $g(\tilde{\lambda})$. Given any $\gamma \in(0, \beta)$ and $M>0$ we partition the state space into four regions as follows. We let

$$
\begin{gathered}
S_{\gamma, M}=\left\{(N, \hat{N}): N \geq M \text { or } \hat{N} \geq M, \beta-\gamma \leq \frac{N}{\hat{N}} \leq 1+\gamma\right\}, \\
R_{\gamma, M}^{-}=\left\{(N, \hat{N}): \hat{N} \geq M, \frac{N}{\hat{N}}<\beta-\gamma\right\}, \\
R_{\gamma, M}^{+}=\left\{(N, \hat{N}): N \geq M, \frac{N}{\hat{N}}>1+\gamma\right\}, \\
Q_{M}=\{(N, \hat{N}): N<M, \hat{N}<M\} .
\end{gathered}
$$

We also let $R_{\gamma, M}=R_{\gamma, M}^{-} \cup R_{\gamma, M}^{+}$.

Lemma 3.3: There exist some $M>0, \gamma>0, \delta>0$, such that $5 \gamma<\beta$ and

$$
\begin{array}{ll}
c(N, \hat{N}) \leq-\delta, & \forall(N, \hat{N}) \in S_{5 \gamma, M}, \\
d(N, \hat{N}) \leq-\hat{o}, & \forall(N, \hat{N}) \in R_{\gamma, M}^{-},
\end{array}
$$




$$
d(N, \hat{N}) \geq \delta, \quad \forall(N, \hat{N}) \in R_{\gamma, M}^{+}
$$

Proof:Notice that $\lambda-\alpha e^{-\alpha}$ is negative when $\alpha=1$ (because $\lambda<e^{-1}$ ) as well as when $\alpha=\beta$ (because of Lemma 3.2(iii)) and is monotonic in between. Furthermore, it is a continuous function of $\alpha$ and therefore there exist $\gamma>0$ and $\delta_{1}>0$ such that $\lambda-\alpha e^{-\alpha} \leq-\delta_{1}, \forall \alpha \in[\beta-5 \gamma, 1+5 \gamma]$. Hence, using Lemma 3.1, $c(N, \hat{N}) \leq-\delta_{1}+h(M) \leq-\frac{\delta_{1}}{2}, \forall(N, \hat{N}) \in S_{5 \gamma, M}$, provided that we take $M$ large enough so that $h(M) \leq \frac{\delta_{1}}{2}$. This proves (3.4) and fixes our choice of $\gamma$. For inequalities (3.5) and (3.6), we use the strict monotonicity of $f$ to conclude that $f(\alpha, \tilde{\lambda}) \leq f(\beta-\gamma, \tilde{\lambda})<0$, $\forall \alpha \leq \beta-\gamma$ and $f(\alpha, \tilde{\lambda}) \geq f(1+\gamma, \tilde{\lambda})>0, \forall \alpha \geq 1+\gamma$. The desired result follows again by choosing $M$ large enough and using Lemma 3.1.

From now on we assume that $M$ and $\gamma$ have been fixed and that inequalities (3.4)-(3.6) hold. We introduce a Lyapunov function which exploits the properties of the drift of $\left(N_{t}, \hat{N}_{t}\right)$ in the regions we introduced earlier. Namely, we let

$$
V(N, \hat{N})=\max \left\{N, \frac{1+3 \gamma}{3 \gamma}(N-\hat{N}), \frac{\beta-3 \gamma}{1-\beta+3 \gamma}(\hat{N}-N)\right\}
$$

and notice that the first, second and third expression inside the brackets becomes effective when $N$ belongs to $S_{3 \gamma, M}, R_{3 \gamma, M}^{+}, R_{3 \gamma, M}^{-}$, respectively. Unfortunately, for any $\Delta>0$, the inequality $E\left[V\left(N_{t+1}, \hat{N}_{t+1}\right) \mid\left(N_{t}, \hat{N}_{t}\right)\right] \leq V\left(N_{t}, \hat{N}_{t}\right)-\Delta$ fails to hold at the boundary between adjacent regions. However, we will show below that, if $J$ is chosen large enough, then there exists some $\Delta>0$ such that

$$
E\left[V\left(N_{t+J}, \hat{N}_{t+J}\right) \mid\left(N_{t}, \hat{N}_{t}\right)=(N, \hat{N})\right] \leq V(N, \hat{N})-\Delta . \quad \forall(N, \hat{N}) \notin Q_{M+J^{2}} .
$$

Our method consists of estimating the decrease in $V$ by separately considering likely and unlikely events, starting with unlikely ones. Given some integer $J$ and some $t \geq 0$, we define a stopping time $\tau_{J}$ by $\tau_{J}=\min \left\{s \geq t: \sum_{k=t}^{b} A_{k} \geq J\right\}$, where $A_{k}$ is the number of new packets generated at time $k$. We then have the following two auxiliary results whose proof is straightforward (using, for example, the same methods as in the proof of Proposition 2.2) and is omitted:

$$
\begin{gathered}
\lim _{J \rightarrow \infty} J P\left(\tau_{J} \leq J\right)=0 \\
\lim _{J \rightarrow \infty} E\left[c_{1} J+c_{2} \sum_{k=t}^{t+J} A_{k} ; \tau_{J} \leq J\right]=0, \quad \forall c_{1}, c_{2} .
\end{gathered}
$$

We notice that $\left|N_{t+1}-N_{t}\right| \leq 1+A_{t}$ and $\left|\hat{N}_{t+1}-\hat{N}_{t}\right| \leq \frac{1}{e-2} \leq 2+\hat{\lambda} \leq 3$. It then follows from (3.7) that

$$
\left|V\left(N_{t+1}, \hat{N}_{t+1}\right)-V\left(N_{t}, \hat{N}_{t}\right)\right| \leq \max \left\{1, \frac{1+3 \gamma}{3 \gamma}, \frac{\beta-3 \gamma}{1-\beta+3 \gamma}\right\}\left(4+A_{t}\right) \leq C\left(1+A_{t}\right),
$$


for some constant $C$. Therefore, there exists some $C$, independent of $J$, such that

$$
\left|V\left(N_{t+1}, \hat{N}_{t+1}\right)-V\left(N_{t}, \hat{N}_{t}\right)\right| \leq C J+C \sum_{k=t}^{t+J} A_{t}
$$

Using (3.10), we see that if $J$ is chosen large enough, then

$$
E\left[V\left(N_{t+J}, \hat{N}_{t+J}\right)-V\left(N_{t}, \hat{N}_{t}\right) ; \tau_{J} \leq J \mid\left(N_{t}, \hat{N}_{t}\right)\right]
$$

can be made as close to zero as desired.

We now consider the event $\tau_{J}>J$.

Lemma 3.4: $J$ can be chosen large enough so that, if $\tau_{J}>J$, then the following are true:

(i) If $X_{t} \in S_{2 \gamma, M+J^{2}}$, then $X_{t+k} \in S_{3 \gamma, M}, \forall k \in[0, J]$.

(ii) If $X_{t} \in S_{4 \gamma, M+J^{2}} \cap R_{2 \gamma, M+J^{2}}^{+}$, then $X_{t+k} \in S_{5 \gamma, M} \cap R_{\gamma, M}^{+}, \forall k \in[0, J]$.

(iii) If $X_{t} \in R_{4 \gamma, M+J^{2}}^{+}$, then $X_{t+k} \in R_{3 \gamma, M}^{+}, \forall k \in[0, J]$.

(iv) Statements (ii) and (iii) remain true if we replace $R^{+}$by $R^{-}$.

Proof: If $\tau_{J}>J$, then $\left|N_{t+k}-N_{t}\right| \leq 3 J$ and $\left|\hat{N}_{t+k}-\hat{N}_{t}\right| \leq 2 J, \forall k \in[0, J]$. On the other hand, notice that the distance between $S_{2 \gamma, M+J^{2}}$ and the complement of $S_{3 \gamma, M}$ is of the order of $J^{2}$ and part (i) follows. The proof is similar for the remaining parts of the Lemma and is omitted.

From now on, we assume that $J$ is large enough so that the statements of Lemma 3.4 hold. We start by considering the case $\left(N_{t}, \hat{N}_{t}\right) \in S_{2 \gamma, M+J^{2}}$. Then, $V\left(N_{t}, \hat{N}_{t}\right)=N_{t}$. If, in addition, $\tau_{J}>J$, then $\left(N_{t+k}, \hat{N}_{t+k}\right) \in S_{3 \gamma, M+J^{2}}, \forall k \in[0, J]$ (by Lemma 3.4) and $V\left(N_{t+J}, \hat{N}_{t+J}\right)=N_{t+J}$. Thus, using (3.4) and assuming that $J$ is large enough so that $P\left(\tau_{j}>J\right)>\frac{1}{2}$ (which is possible, due to (3.9)) we obtain

$$
\begin{gathered}
E\left[V\left(N_{t+J}, \hat{N}_{t+J}\right)-V\left(N_{t}, \hat{N}_{t}\right) ; \tau>J_{J} \mid\left(N_{t}, \hat{N}_{t}\right)\right]=E\left[N_{t+J}-N_{t} ; \tau_{J}>J \mid\left(N_{t}, \hat{N}_{t}\right)\right]= \\
\sum_{k=0}^{J-1} E\left[c\left(N_{t+k}, \hat{N}_{t+k}\right) ; \tau_{J}>J \mid\left(N_{t}, \hat{N}_{t}\right)\right] \leq-\delta J P\left(\tau_{J}>J\right) \leq-\frac{\delta J}{2},
\end{gathered}
$$

where $\delta>0$ is the constant of Lemma 3.3.

Next we consider the case $\left(N_{t}, \hat{N}_{t}\right) \in R_{5 \gamma, M+J^{2}}^{+}$. The same argument as above yields, for $J$ large enough,

$$
\begin{gathered}
E\left[V\left(N_{t+J}, \hat{N}_{t+J}\right)-V\left(N_{t}, \hat{N}_{t}\right) ; \tau_{J}>J \mid\left(N_{t}, \hat{N}_{t}\right)\right]=\frac{1+3 \gamma}{3 \gamma} E\left[-\tilde{N}_{t+J}+\tilde{N}_{t} ; \tau_{J}>J \mid\left(N_{t}, \hat{N}_{t}\right)\right]= \\
-\frac{1+3 \gamma}{3 \gamma} \sum_{k=0}^{J-1} E\left[d\left(N_{t+k}, \hat{N}_{t+k}\right) ; \tau_{J}>J \mid\left(N_{t}, \hat{N}_{t}\right)\right] \leq-\frac{1+3 \gamma}{3 \gamma} \frac{\delta J}{2} .
\end{gathered}
$$

A similar argument applies to the case where $X_{t} \in R_{5 \gamma, M+J^{2}}^{-}$. 
We now consider the slightly more complicated case where $\left(N_{t}, \hat{N}_{t}\right) \in S_{4 \gamma, M+J^{2}}$ and $\left(N_{t}, \hat{N}_{t}\right) \notin$ $S_{2 \gamma, M+J^{2}}$. There are two subcases to consider: a) $\frac{N}{\hat{N}} \in(1+2 \gamma, 1+4 \gamma]$ and b) $\frac{N}{\hat{N}} \in[\beta-4 \gamma, \beta-2 \gamma)$. We only consider the first subcase, since the argument for the second one is identical. We therefore have $V\left(N_{t}, \hat{N}_{t}\right)=\max \left\{N,-\frac{1+3 \gamma}{3 \gamma} \tilde{N}_{t}\right\}$. Furthermore, if $\tau_{J}>J$, then $\left(N_{t+k}, \hat{N}_{t+k}\right)$ stays inside $S_{5 \gamma, M} \cap R_{\gamma, M}^{+}, \forall k \in[0, J]$. Thus, $V\left(N_{t+J}, \hat{N}_{t+J}\right)=\max \left\{N_{t+J},-\frac{1+3 \gamma}{3 \gamma} \tilde{N}_{t+J}\right\}$. Consequently,

$$
\begin{gathered}
E\left[V\left(N_{t+J}, \hat{N}_{t+J}\right)-V\left(N_{t}, \hat{N}_{t}\right) ; \tau_{J}>J \mid\left(N_{t}, \hat{N}_{t}\right)\right] \leq \\
E\left[\max \left\{N_{t+J}-N_{t},-\frac{1+3 \gamma}{3 \gamma}\left(\tilde{N}_{t+J}-\tilde{N}_{t}\right)\right\} ; \tau_{J}>J \mid\left(N_{t}, \hat{N}_{t}\right) \leq\right. \\
E\left[\max \left\{0, N_{t+J}-N_{t}+\frac{J \delta}{2}\right\} ; \tau_{J}>J \mid\left(N_{t}, \hat{N}_{t}\right)\right]+ \\
+\frac{1+3 \gamma}{3 \gamma} E\left[\max \left\{0,-\tilde{N}_{t+J}+\tilde{N}_{t}+\frac{J \delta}{2} ; \tau_{J}>J \mid\left(N_{t}, \hat{N}_{t}\right)\right]+E\left[-\frac{J \delta}{2} ; \tau_{J}>J \mid\left(N_{t}, \hat{N}_{t}\right)\right] .\right.
\end{gathered}
$$

Here, $\delta$ is the constant of Lemma 3.3 and we have used the inequalities $\max \{a, b\}-\max \{c, d\} \leq$ $\max \{a-c, b-d\}$ and $\max \{a, b\} \leq \max \{0, a+f\}+\max \{0, b+f\}-f$, with $f=\frac{J \delta}{2}$. We consider the first summand in the right hand siide of (3.11). Let $W_{k}=N_{t+k}-N_{t}+\frac{k \delta}{2}$. Clearly, $\left\{W_{k}, \exists_{t+k}\right\}$ is exponential type because $\left|W_{k+1}-W_{k}\right| \leq 1+A_{t+k}$. Furthermore, using Lemma 3.3,

$$
E\left[W_{k+1}-W_{k} ; \tau_{J}>J \mid \mathcal{F}_{k}\right]=E\left[N_{t+k+1}-N_{t+k}+\frac{\delta}{2} ; \tau_{J}>J \mid \xi_{t+k}\right] \leq-\frac{\delta}{2}
$$

Thus, Propcsition 2.2 applies and shows that the first summand in (3.11) is bounded above by some $B$ independent of $J$. The same conciusion is obtained, by an identical argument, for the second summand in (3.11). Finally, the last term in (3.11) is equal to $-\frac{J \delta}{2} P\left(\tau_{J}>J\right)$. Taking $J$ large enough and using (3.9), this term can be made arbitrarily negative. It follows that the right hand side of (3.11) can become negative and bounded away from zero by proper choice of $J$. This concludes the proof of (3.8).

The proof of the theorem may be now completed as follows. Let $G=\max \left\{1, \frac{1+3 \gamma}{3 \gamma}, \frac{\beta-3 \gamma}{1-\beta+3 \gamma}\right\}(M+$ $\left.J^{2}\right)$. Whenever $V\left(N_{t}, \hat{N}_{t}\right) \geq G$, then either $N \geq M+J^{2}$ or $\hat{N} \geq M+J^{2}$ and (3.8) holds. Furthermore $\left\{V\left(N_{t}, \hat{N}_{t}\right), \mathcal{F}_{t}\right\}$ is exponential type. Hence Proposition 2.1 applies and shows that the stopping time $r=\min \left\{k: V\left(N_{k J}, \hat{N}_{k J}\right)<G\right\}$ is exponential type, for any initial state. From this it follows easily that the time until $\left(N_{t}, \hat{N}_{t}\right)$ becomes equal to $(0,1)$ is also exponential type and concludes the proof of the theorem.

Remark: It should be clear from the above proof that it is not necessary to assume that the arrival process $A_{t}$ is Poisson or even that the random variables $A_{t}$ are independent identically distributed. One only needs to assume that $\left\{A_{t}, F_{t}\right\}$ is exponential type, in the sense of Section II.

IV. THE CASE WHERE $\hat{\lambda}<\lambda$. 
With a minor modification of the proof in Section III it can be shown that for any fixed $\lambda<e^{-1}$ there exists some $\epsilon>0$ such that if $|\lambda-\hat{\lambda}|<\epsilon$, then $\left\{X_{t}\right\}$ is geometrically ergodic. In general, however, $\epsilon$ will depend on $\lambda$ and will tend to zero as $\lambda$ approaches $e^{-1}$.

Suppose now that $\lambda$ is very close to $e^{-1}$ and that $\lambda-\hat{\lambda}$ is positive and sufficiently large. Then, $\left\{X_{t}\right\}$ will no longer be ergodic, as indicated by the following argument. If $\lambda \approx e^{-1}$, the only way of having a stable (ergodic) process is to have some mechanism that ensures that the probability of transmission by each station is very close to $1 / N_{t}$, at least whenever $N_{t}$ is large. Equivalently, we want $\frac{N_{t}}{\hat{N}_{t}} \approx 1$. However, when $\tilde{\lambda} \neq 1$, then $\tilde{N}$ drifts away from zero, because $f(1, \tilde{\lambda}) \neq 1$, where $f$, the function defined in (3.3) (which is the approximate drift of $\tilde{N}$, according to Lemma 3.1. Therefore, $X_{t}$ will tend to spend most of its time in a region where $\alpha$ is bounded away from 1 and, consequently the probability of a succesful transmission is bounded away from $e^{-1}$. Instability then results.

One might try to make a similar argument for the case $\lambda<\hat{\lambda}$. In this case the probability of a succesful transmission is again bounded away from $e^{-1}$. However, since $\lambda<\hat{\lambda} \leq e^{-1}$, there is less input traffic to be accommodated and instability does not arise. (This is the essence of part (iii) of Lemma 3.2.)

It is suggested in [1] that $\lambda$ could be estimated on line, if it is unkown. One possible method [1] is to let $\hat{\lambda}_{t}$ be the number of succesful transmissions up to time $t$, divided by $t$. Such an estimator loses its ability to adapt to changes in the input traffic statistics, as time goes to infinity. For this reison an exponential weight was used in [1] to discount old data. It is unclear whether such a method can achieve stably throughput up to $e^{-1}$. Given the result of Section 3 , overestimatin $3 \lambda$ by using the estimate $\hat{\lambda}=e^{-1}$ cannot result to instability and this seems to be a reascnable choice.

\section{REFERENCES.}

1. Rivest, R.L., “Network Control by Bayesian Broadcast”, Technical Report TM-287, Laboratory for Computer Science, Massachusetts Institute of Technology, September 1985.

2. Hajek, B., "Hitting-Time and Occupation-Time Bounds Implied by Drift Analysis with Applications", Advances in Applied Probability, 14, 1982, pp. 502-525.

3. Hajek, B., and Van Loon, T., "Decentralized Dynamic Control of a Multiaccess Broadcast Channel", IEEE Transactions on Automatic Control, AC-27, 3, 1982, pp. 559-569.

4. Kelly, F.P., "Stochastic Models of Computer Communication Systems", J. Royal Statistical Society, B, 47, 1, 1985.

5. Mikhailov, V.A., "Methods of Random Multiple Access", Candidate Eng. Thesis, Moscow Institute of Physics and Technology, 1979. 
ACKNOWLEDGEMENTS: I would like to thank Dimitri Bertsekas and Robert Gallager for suggesting the problem studied here and several discussions, as well as Bruce Hajek who brought the existence of references [4] and [5] to my attention. 